Pisco Ned Lublishing

Original Research Article

\title{
The Core Literacy of Mathematics-The Cultivation of Mathematical Operation Ability
}

\section{Chengfeng Li}

Attached High School of Yunnan Arts University 650031

\begin{abstract}
The core literacy of mathematics can be divided into the following aspects: mathematical abstraction, mathematical thinking, mathematical application, mathematical operation, logical reasoning, data analysis, mathematical modeling, intuitive imagination, number-shape connection, rigorous verification, mathematical emotion, reasonable guessing, Mathematical culture, careful thinking, use of mathematical tools, mathematical language or symbolic language. Mathematical ability is the most basic and main ability, and it is the foundation of other mathematical literacy. Cultivation of Mathematical Ability

Keywords: Core Literacy of Mathematics; Computing Ability
\end{abstract}

\section{The ability to mine topic information}

Fully mining the information implicit in the known conditions and conclusions is a necessary condition for seeking and designing a reasonable and simple calculation method.

example 1

Known inequalities about $\mathrm{x} \log _{2}(4-x)^{2}>\log _{2}(x-a)-1$ The solution set is A,and $A \cap Z=\{0,-1\}$, among themZ $=\{$ Integer $\}$, Try to find the value range of the real number a.

Solution 1: by $\log _{2}(4-x)^{2}>\log _{2}(x-a)-1$ Get: $\log _{2}(4-x)^{2}>\log _{2}\left(\frac{x-a}{2}\right) \Rightarrow\left\{\begin{array}{c}4-x^{2}>\frac{x-a}{2} \\ \frac{x-a}{2}>0\end{array} \Rightarrow\left\{\begin{array}{c}x>a \\ 2 x^{2}+x-(a+8)<0\end{array}\right.\right.$

$\because A \cap Z=\{0,-1\}$

$\therefore\left\{\begin{array}{l}\Delta=1^{2}+4 \times 2 \times(8+a)>0 \\ a \leq-\frac{1}{4}-\frac{\sqrt{65+8 a}}{4}<-1 \\ -2 \leq-\frac{1}{4}-\frac{\sqrt{65+8 a}}{4} \leq-1 \\ 0<-\frac{1}{4}+\frac{\sqrt{65+8 a}}{4} \leq 1\end{array}\right.$

$\therefore\left\{\begin{aligned} & \Delta=1^{2}+4 \times 2 \times(8+a)>0 \\ &-\frac{1}{4}-\frac{\sqrt{65+8 a}}{4}<a<-\frac{1}{4}+\frac{\sqrt{65+8 a}}{4} \\ &-2 \leq a<-1 \\ & 0<-\frac{1}{4}+\frac{\sqrt{65+8 a}}{4} \leq 1\end{aligned}\right.$

then: $-7 \leq a<-5$

This is an open-access article distributed under the terms of the Creative Commons Attribution Non-Commercial License (http://creativecommons.org/licenses/by-nc/4.0/), which permits non-commercial use, distribution, and reproduction in any medium, provided the original work is properly cited. 
Solution 2: by $\log _{2}(4-x)^{2}>\log _{2}(x-a)-1$ Get $\log _{2}(4-x)^{2}>\log _{2} \frac{x-a}{2}$ then $\left\{\begin{array}{c}4-x^{2}>\frac{x-a}{2} \\ \frac{x-a}{2}>0\end{array}\right.$ which is $\left\{\begin{array}{c}2 x^{2}+x-(8+a)<0 \\ x>a\end{array}\right.$ $\because A \cap Z=\{0,-1\}$

$$
\begin{gathered}
2 \times 0^{2}+0-(8+\alpha)<0 \\
2 \times(-2)^{2}+(-1)-(8+\alpha)<0 \\
\alpha<0 \\
\alpha<-1 \\
2 \times(-2)^{2}+(-2)-(8+\alpha) \geq 0 \text { or } \alpha \geq-2 \\
2 \times 1^{2}+1-(8+\alpha) \geq 0 \text { or } \alpha \geq 1
\end{gathered}
$$

Get: $-7 \leq a<-5$

From the solution process of solution 1 and solution 2, solution 1, Information $A \cap Z=\{0,-1\}$ Digging only stays at $A \cap Z=\{0,-1\}$, Explain that the value of the left endpoint of set $\mathrm{A}$ is $[-2,-1)$ between, The value of the right endpoint is $(0,1]$ between, Strive for A。 when $\Delta=1^{2}+4 \times 2 \times(8+a)>0$ And $a \leq-\frac{1}{4}-\frac{\sqrt{65+8 a}}{4}$ Time, $\mathrm{A}=\left(-\frac{1}{4}-\frac{\sqrt{65+8 a}}{4},-\frac{1}{4}+\frac{\sqrt{65+8 a}}{4}\right)$;

when $\Delta=1^{2}+4 \times 2 \times(8+a)>0$ And $\frac{-1-\sqrt{65+8 a}}{4}<a<\frac{-1+\sqrt{65+8 a}}{4}$ Time, $\mathrm{A}=\left(a, \frac{-1+\sqrt{65+8 a}}{4}\right)$, Thus a set of inequalities about a is established. And solution 2, Information $A \cap Z=\{0,-1\}$ inferred:0 with -1 Inequality group $\left\{\begin{array}{c}2 x^{2}+x-(8+a)<0 \\ x>a\end{array}(*)\right.$ Elements in the solution set, And -2 and 1 are not elements in the solution set of this inequality group $(*)$,

Therefore: $2 \times 0^{2}+0-(8+\alpha)<0$

$$
\begin{gathered}
2 \times(-2)^{2}+(-1)-(8+\alpha)<0 \\
2 \times(-2)^{2}+(-2)-(8+\alpha) \geq 0 \text { or } \alpha \geq-2 \\
2 \times 1^{2}+1-(8+\alpha) \geq 0 \text { or } \alpha \geq 1
\end{gathered}
$$

It can be seen that the ability to mine topic information is an important part of computing power.

\section{Ability to use definitions, theorems, formulas and rules}

Operation refers to finding the result of an arithmetic problem or formula according to the laws of mathematics. 
When calculating the same arithmetic problem, the basis used is different, resulting in a different degree of complexity in the calculation process.

\section{Enhance students' concept of reflecting on the calculation process}

To improve students' arithmetical abilities, students are required to not only know "what" or "why" when mastering knowledge, but also to know "what is it for" and "how to use". Only the knowledge learned can be used effectively When solving problems, it is considered "effective" acquisition. Only in the process of reflection and calculation, students can "see through the phenomenon and see the essence" and be more profound and precise.

\section{References}

1. "Hubei Education • Education" (2020 s) Author: Fan Zhe.

2. "Mathematics New Curriculum Standards" (2019 edition).

3. "Mathematics" (high school textbook) People's Education Press.

4. "Five Years of College Entrance Examination • Three Years of Simulation" (College Entrance Examination Writing Number, 2019B Edition). 